\title{
NOTE ON THE EQUATION OF HEAT CONDUCTION*
}

\author{
BY A. E. HEINS
}

1. Introduction. In recent years, operational methods in mathematics have been a subject of much discussion. Heaviside, in his papers, by somewhat artificial methods, succeeded in solving a number of differential equations, especially those common to the electro-magnetic theory. The newer developments in operational calculus make no attempt to follow Heaviside's methods. More recent literature shows that the Fourier integral (Jeffreys, $\uparrow$ Bush $\ddagger$ ) plays an important role in these methods. In a thesis of Levinson, $\S$ it was demonstrated that the Fourier transform could be employed to even better advantage than the Fourier integral.

In this note, it is proposed to quote the Fourier transform theorem of several variables, and apply a particular form of it to the solution of the equation for the flow of heat in three dimensions.

2. Fourier Transform of Several Variables. Here we shall consider a function of $k$ real variables $F\left(x_{1}, \cdots, x_{k}\right)$ in a closed domain $a_{\lambda} \leqq x_{\lambda} \leqq b_{\lambda},(\lambda=1, \cdots, k)$, capable of taking on complex values. Consider an integral with infinite limits, such as

$$
\int_{-\infty}^{\infty} \cdots \int_{-\infty}^{\infty} F\left(x_{1}, \cdots, x_{k}\right) d x_{1} \cdots d x_{k} .
$$

Such an integral is said to be convergent if the limit of the integral

$$
\begin{array}{r}
\lim _{A_{\lambda} \rightarrow \infty, B_{\lambda} \rightarrow \infty} \int_{-B_{1}}^{A_{1}} \cdots \int_{-B_{k}}^{A_{k}} F\left(x_{1}, \cdots, x_{k}\right) d x_{1} \cdots d x_{k}, \\
(\lambda=1, \cdots, k),
\end{array}
$$

* From a thesis presented for the degree Master of Science at the Massachusetts Institute of Technology, Oct. 30, 1934, under the title Applications of the Fourier transform theorem. Presented to the Sosiety, December 28, 1934.

$\dagger \mathrm{H}$. Jeffreys, Operational Methods in Mathematical Physics, Cambridge Tract No. 23, 1931.

‡ V. Bush, Operational Circuit Analysis, 1929.

$\S$ N. Levinson, Applications of the Fourier integral, master's thesis, May, 1934, Massachusetts Institute of Technology, unpublished as yet. 
is determined. If, moreover,

$$
\int_{-\infty}^{\infty}\left|F\left(x_{1}, \cdots, x_{k}\right)\right| d x_{\lambda}<\infty, \quad(\lambda=1, \cdots, k),
$$

then $F\left(x_{1}, \cdots, x_{k}\right)$ is called absolutely integrable.

Let there be given a function $F\left(x_{1}, \cdots, x_{k}\right)$ with the following properties:

1. It has only a finite number of finite discontinuities.

2. It is piecewise continuous.

3. It is absolutely integrable and bounded.

Then under these hypotheses the Fourier integral of the function $F\left(x_{1}, \cdots, x_{k}\right)$ exists and is given by the following expression :

$$
\begin{aligned}
& (2 \pi)^{k} F\left(x_{1}, \cdots, x_{k}\right) \\
& =\int_{-\infty}^{\infty} \cdots \int_{-\infty}^{\infty} F\left(t_{1}, \cdots, t_{k}\right) e^{-i \Sigma_{\lambda=1}^{k}\left[u_{\lambda}\left(t_{\lambda}-x_{\lambda}\right)\right]} d t_{1} d u_{1} \cdots d t_{k} d u_{k} .
\end{aligned}
$$

By rearranging this integral, the Fourier transform theorem is obtained. Denote the integral

$$
\left(\frac{1}{2 \pi}\right)^{k / 2} \int_{-\infty}^{\infty} \cdots \int_{-\infty}^{\infty} F\left(x_{1}, \cdots, x_{k}\right) e^{-i \sum_{\lambda=1}^{k} t_{\lambda} x_{\lambda}} d x_{1} \cdots d x_{k}
$$

by $g\left(t_{1}, \cdots, t_{k}\right)$. Then we have

$$
(2 \pi)^{k / 2} F\left(x_{1}, \cdots, x_{k}\right)=\int_{-\infty}^{\infty} \cdots \int_{-\infty}^{\infty} g\left(t_{1}, \cdots, t_{k}\right) e^{i \sum_{\lambda=1}^{k} t_{\lambda} x_{\lambda}} d t_{1} \cdots d t_{k} .
$$

That is, $g\left(t_{1}, \cdots, t_{k}\right)$ is the transform of $F\left(x_{1}, \cdots, x_{k}\right)$ and, reciprocally, $F\left(x_{1}, \cdots, x_{k}\right)$ is the transform of $g\left(t_{1}, \cdots, t_{k}\right)$. This dual character of the Fourier transform theorem plays an important role in the solution of certain partial differential equations.

In the particular problem treated here, we make use of a special form of the transform theorem, commonly called the Mellin transform. To bring the Fourier transform into this form, consider first a function $F_{1}\left(x_{1}, \cdots, x_{k}\right)$ given by

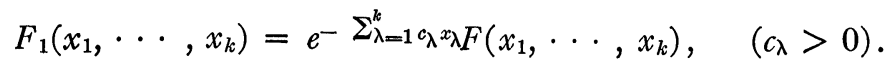


Then the Fourier integral for $F_{1}\left(x_{1}, \cdots, x_{k}\right)$ becomes $\left(\frac{1}{2 \pi}\right)^{k} \int_{-\infty}^{\infty} \cdots \int_{-\infty}^{\infty} e^{-i \sum_{\lambda=1}^{k}\left[u_{\lambda}\left(t_{\lambda}-x_{\lambda}\right)-i c_{\lambda} t_{\lambda}\right]} F_{1}\left(t_{1}, \cdots, t_{k}\right) d t_{1} d u_{1} \cdots d t_{k} d u_{k}$.

Let $u_{\lambda}=v_{\lambda}+i c_{\lambda}$. Then $F_{1}\left(x_{1}, \cdots, x_{k}\right)$ is

$$
\left(\frac{1}{2 \pi}\right)^{k} \int_{-i c_{1}-\infty}^{-i c_{1}+\infty} \int_{-\infty}^{\infty} \cdots \int_{-\infty}^{\infty} e^{i \sum_{\lambda=1}^{k}\left[\left(v_{\lambda}+i c_{\lambda}\right) x_{\lambda}\right]} \psi d t_{1} d v_{1} \cdots d t_{k} d v_{k},
$$

where $\psi$ is

$$
F_{1}\left(t_{1}, \cdots, t_{k}\right) e^{-\sum_{\lambda=1}^{k} c^{c} t_{\lambda}} e^{-i \sum_{\lambda=1}^{k}\left(v_{\lambda}+i c_{\lambda}\right) t_{\lambda}},
$$

or the value of $F\left(x_{1}, \cdots, x_{k}\right)$ is

$$
\begin{aligned}
& \left(\frac{1}{2 \pi}\right)^{k} \int_{-i c_{1}-\infty}^{-i c_{1}+\infty} \int_{-\infty}^{\infty} \cdots \\
& \int_{-\infty}^{\infty} F\left(t_{1}, \cdots, t_{k}\right) e^{-i \sum_{\lambda=1}^{k} v_{\lambda}\left(t_{\lambda}-x_{\lambda}\right)} d t_{1} d v_{1} \cdots d t_{k} d v_{k} .
\end{aligned}
$$

Now put $p_{\lambda}=i v_{\lambda}$ and we obtain for $F\left(x_{1}, \cdots, x_{k}\right)$ the value

$\left(\frac{1}{2 \pi i}\right)^{k} \int_{-i \infty+c_{1}}^{i \infty+c_{1}} \int_{-\infty}^{\infty} \cdots \int_{-\infty}^{\infty} F\left(t_{1}, \cdots, t_{k}\right) e^{-\Sigma_{\lambda=1}^{k} p_{\lambda}\left(t_{\lambda}-x_{\lambda}\right)} d t_{1} d p_{1} \cdots d t_{k} d p_{k}$.

If this integral is split into two parts as the Fourier integral was, the Mellin transform is obtained. That is,

$g\left(p_{1}, \cdots, p_{k}\right)=\int_{-\infty}^{\infty} \cdots \int_{-\infty}^{\infty} F\left(t_{1}, \cdots, t_{k}\right) e^{-\Sigma_{\lambda=1}^{k} p_{\lambda} t_{\lambda}} d t_{1} \cdots d t_{k}$,

and

$$
\begin{aligned}
F\left(x_{1}, \cdots, x_{k}\right)=\left(\frac{1}{2 \pi i}\right)^{k} \int_{-i \infty+c_{1}}^{i \infty+c_{1}} & \\
& \cdots \int_{-i \infty+c_{k}}^{i \infty+c_{k}} g\left(p_{1}, \cdots, p_{k}\right) e^{\Sigma_{\lambda=1}^{k} p_{\lambda} x_{\lambda}} d p_{1} \cdots d p_{k} .
\end{aligned}
$$

In physical problems we require oftentimes no knowledge of the past state of the system. That is, we are only interested in everything which takes place when $t$ (time) $\geqq 0$. It is for this reason that it is of convenience to arrange coordinates such that 
$F\left(x_{1}, \cdots, x_{k}\right)=0$ for all $x_{\lambda}<0$. Then $g\left(p_{1}, \cdots, p_{k}\right)$ becomes under this restriction

$$
\int_{0}^{\infty} \cdots \int_{0}^{\infty} F\left(t_{1}, \cdots, t_{k}\right) e^{-\Sigma_{\lambda=1}^{k} p_{\lambda} t_{\lambda}} d t_{1} \cdots d t_{k},
$$

since the interval $(-\infty, 0)$ does not contribute anything to this transform.

There is just one more theorem which is of interest. Given $f_{1}\left(x_{1}, \cdots, x_{k}\right)$ and $f_{2}\left(x_{1}, \cdots, x_{k}\right)$ and their respective transforms $g_{1}\left(p_{1}, \cdots, p_{k}\right)$ and $g_{2}\left(p_{1}, \cdots, p_{k}\right)$, what is the transform of $g_{1}\left(p_{1}, \cdots, p_{k}\right) g_{2}\left(p_{1}, \cdots, p_{k}\right)$ ? This is answered by the faltung theorem. That is, if the function $f_{1}\left(x_{1}, \cdots, x_{k}\right)$ is the transform of $g_{1}\left(p_{1}, \cdots, p_{k}\right)$ and the function $f_{2}\left(x_{1}, \cdots, x_{k}\right)$ is the transform of $g_{2}\left(p_{1}, \cdots, p_{k}\right)$, then the transform of the function $g_{1}\left(p_{1}, \cdots, p_{k}\right) g_{2}\left(p_{1}, \cdots, p_{k}\right)$ is given by

$$
\begin{aligned}
f\left(x_{1}, \cdots, x_{k}\right)= & \int_{0}^{x_{1}} \cdots \int_{0}^{x_{k}} f_{1}\left(y_{1}, \cdots, y_{k}\right) \\
& \cdot f_{2}\left(x_{1}-y_{1}, \cdots, x_{k}-y_{k}\right) d y_{1} \cdots d y_{k} .
\end{aligned}
$$

We must remark that this theorem holds under the restriction that $f_{2}\left(x_{1}-y_{1}, \cdots, x_{k}-y_{k}\right)=0$ for $y_{\lambda}>x_{\lambda}$ and $f_{1}\left(y_{1}, \cdots, y_{k}\right)=0$ for $y_{\lambda}<0 . *$

3. Heat Flow in an Infinite Medium. We propose to solve here the following linear parabolic differential equation

$$
K\left(\frac{\partial^{2} V}{\partial x^{2}}+\frac{\partial^{2} V}{\partial y^{2}}+\frac{\partial^{2} V}{\partial z^{2}}\right)=\frac{\partial V}{\partial t} .
$$

Multiply equation (1) by $e^{-p_{1} x-p_{2} y-p_{3} z}$ and integrate from 0 to $\infty$ over $x, y$, and $z$. Then we find by the transform theorem

$$
\frac{\partial g}{\partial t}=K\left(p_{1}^{2}+p_{2}^{2}+{p_{3}^{2}}^{2}\right) g
$$

Thus we see that $p_{1}, p_{2}, p_{3}$ act as partial derivatives in the sense that $p_{1}$ acting on $g$ is the transform of $\partial V / \partial x$. Similarly $p_{1}{ }^{2}$

* See S. Bochner, Vorlesungen über Fouriersche Integrale, 1932, for a complete discussion of the Fourier transform. 
acting on $g$ is the transform of $\partial^{2} V / \partial x^{2}$. Solving equation (2) for $g$ we get

$$
g=A e^{k t\left(p_{1}{ }^{2}+p_{2}{ }^{2}+p_{3}{ }^{2}\right)},
$$

where $A$ is a function of $p_{1}, p_{2}, p_{3}$ to be determined.

For initial conditions we put the original temperature distribution equal to $f(x, y, z)$ when $t=0$. Moreover, without any lack of generality, we can limit the flow to the positive octant so that $V=0$ for $x<0, y<0, z<0$. Let $H\left(p_{1}, p_{2}, p_{3}\right)$ be the transform of $f(x, y, z)$. Then $A\left(p_{1}, p_{2}, p_{3}\right)=H\left(p_{1}, p_{2}, p_{3}\right)$ and

$$
\begin{array}{r}
V=\left(\frac{1}{2 \pi i}\right) \int_{-i \infty+c_{1}}^{i \infty+c_{1}} \int_{-i \infty+c_{2}}^{i \infty+c_{2}} \int_{-i \infty+c_{3}}^{i \infty+c_{3}} H e^{k\left(p_{1}{ }^{2}+p_{2}{ }^{2}+p_{3}{ }^{2}\right) t} \\
\cdot e^{p_{1} x+p_{2} y+p_{3} z} d p_{1} d p_{2} d p_{3} .
\end{array}
$$

To evaluate this expression, consider the transform of

$$
e^{k t\left(p_{1}{ }^{2}+p_{2}{ }^{2}+p_{3}{ }^{2}\right)} \text {. }
$$

That is, we must evaluate

$$
\begin{aligned}
\left(\frac{1}{2 \pi i}\right)^{3} \int_{-i \infty+c_{1}}^{i \infty+c_{1}} e^{k t\left(p_{1}{ }^{2}+p_{1} x /(k t)\right)} d p_{1} \int_{-i \infty+c_{2}}^{i \infty+c_{2}} e^{k t\left(p_{2}{ }^{2}+p_{2} y /(k t)\right)} d p_{2} \\
\\
\cdot \int_{-i \infty+c_{3}}^{i \infty+c_{3}} e^{k t\left(p_{3}{ }^{2}+p_{3} z /(k t)\right)} d p_{3} .
\end{aligned}
$$

This integral may be considered as the product of three integrals, for example,

$$
\left(\frac{1}{2 \pi i}\right) \int_{-i \infty+c_{1}}^{i \infty+c_{1}} e^{k t\left(p_{1} 2+p_{1} x /(k t)\right)} d p_{1} .
$$

This may be written as

$$
\frac{e^{-x^{2} /(4 k t)}}{2 \pi i} \int_{-i \infty+c_{1}}^{i \infty+c_{1}} e^{k t\left(p_{1}+x /(2 k t)\right)^{2}} d p_{1} .
$$

If we put $i u_{1}=(k t)^{1 / 2}\left(p_{1}+x /(2 k t)\right)$, then equation (5) becomes

$$
\frac{e^{-x 2 /(4 k t)}}{2 \pi(k t)^{1 / 2}} \int_{-\infty+i B}^{\infty+i B} e^{-u_{1}^{2}} d u_{1},
$$

where the $B$ 's include variables which do not depend on our 
integration variable. The integral in equation (6) is taken along a path parallel to the $x$ axis. This contour may be deformed and the integral can be taken along the entire $x$ axis. Then equation (6) goes over into

$$
\frac{e^{-x^{2} /(4 k t)}}{2 \pi(k t)^{1 / 2}} \int_{-\infty}^{\infty} e^{-u_{1}^{2}} d u_{1}
$$

This integral is the error integral and its value is $\pi^{1 / 2}$. Hence the product of three such integrals is

$$
\frac{e^{-\left(x^{2}+y^{2}+z^{2}\right) /(4 k t)}}{(4 \pi k t)^{3 / 2}} \text {. }
$$

Applying the faltung theorem to equation (4), we find

$$
\begin{array}{r}
V(x, y, z, t)=\left(\frac{1}{4 \pi k t}\right)^{3 / 2} \int_{0}^{x} \int_{0}^{y} \int_{0}^{z} f\left(x-x_{1}, y-y_{1}, z-z_{1}\right) \\
\cdot e^{-\left(x_{1}{ }^{2}+y_{1}{ }^{2}+z_{1}{ }^{2}\right) /(4 k t)} d x_{1} d y_{1} d z_{1} .
\end{array}
$$

This is the standard solution of this problem, which is usually obtained by separating variables.*

Massachusetts Institute of Technology

* E. Goursat, Cours d'Analyse, vol. 3, p. 107, 1927. 